\title{
Nanoparticle preparation of mefenamic acid by electrospray drying
}

\begin{abstract}
Nanoparticles preparation of Mefenamic acid (MA) by using an electrospray drying method was conducted in this study. Electrospray drying is a process that uses electrostatic force to disperse a conductive liquid stream into fine charged droplets through the coulomb fission of charges in the liquid and finally dry into fine particles. Electrospray drying modes operation usually in Taylor cone jet, and it was formed by controlling applied voltage and liquid flow rate. A conductive liquid (2.77ї 8.55عScmī 1) which is MA solution was prepared by using acetone with concentration 0.041 and $0.055 \mathrm{M}$ before pumping at a flow rate of $3 \ddot{~} 6 \mathrm{ml} / \mathrm{h}$. By applying the applied voltage at $1.3 \ddot{1} 1.5 \mathrm{kV}$, Taylor cone jet mode was formed prior to the electrospray. During electrospray drying process, solvent evaporation from the droplet was occurring that leads to coulomb disruption and may generate to nanoparticles. The dried nanoparticles were collected on a grounded substrate that was placed at varying distance from the electrospray. MA particle with size range of $100 \mathrm{i} 400 \mathrm{~nm}$ were produced by electrospray drying process. Characterization of particles by using X-ray diffractometry (XRD) and differential scanning calorimetry (DSC) show that particles formed into polymorph I.
\end{abstract}

Keyword: Coulomb fission; Electrospray drying; Nanoparticles 